\title{
Cómo argumentar con coherencia ${ }^{8}$
}

\author{
(How to argue with coberence)
}

\author{
José Ángel GascóN*
}

Centro de Educación en Investigación y Desarrollo (CIEDE)

Universidad Católica de la Santísima Concepción (UCSC)

\begin{abstract}
RESUMEN: Cuando hay desacuerdos sobre la calidad de los argumentos, los argumentadores con frecuencia se apoyan en la coherencia. La coherencia argumentativa se manifiesta principalmente en acusaciones de incoherencia y en la presentación de analogías. Con la ayuda de los elementos de la garantía y de las salvedades del modelo de Toulmin, es posible proporcionar un primer análisis de esta noción.
\end{abstract}

PALABRAS CLAVE: argumentación, analogías, garantía, lógica informal, salvedad.

ABSTRACT: When disagreements arise about the quality of arguments, arguers frequently rely on coherence. Argumentative coherence is mainly manifested in accusations of incoherence and in the production of analogies. With the help of the elements of warrant and of rebuttals in Toulmin's model, it is possible to give a first analysis of this notion.

KEYWORDS: argumentation, analogies, informal logic, rebuttal, warrant.

\& Una versión previa de este trabajo fue presentada en el IX Congreso de la Sociedad de Lógica, Metodología y Filosofía de la Ciencia en España. Debo agradecer a los asistentes sus comentarios críticos e incisivos, que me ayudaron a mejorar el artículo. Estoy en deuda especialmente con Paula Olmos, Hubert Marraud, Luis Vega, Javier Vilanova y Enrique Alonso. Agradezco también a dos revisores anónimos de Theoria sus esfuerzos por ayudarme a reforzar varios puntos del artículo. Este trabajo fue posible gracias al proyecto CONICYT/FONDECYT de Postdoctorado nº 3190149 del gobierno de Chile.

\footnotetext{
* Correspondencia a/Correspondence to:: José Ángel Gascón. Alonso de Ribera, 2850. Centro de Educación en Investigación y Desarrollo (CIEDE). Universidad Católica de la Santísima Concepción (UCSC). Concepción (Chile) - jgascon@ucsc.cl - https://orcid.org/0000-0001$5571-660$
}

Cómo citar/How to cite: Gascón, José Ángel (2020). "Cómo argumentar con coherencia»; Theoria. An International Journal for Theory, History and Foundations of Science, 35(3), 327-344. (https://doi.org/10.1387/theoria.20435).

Recibido/Received: 03 diciembre, 2018; Versión final/Final version: 21 agosto, 2019.

ISSN 0495-4548 - elSSN 2171-679X / (C) 2020 UPV/EHU

(c) (i) Esta obra está bajo una licencia

Creative Commons Atribution 4.0 Internacional License 


\section{Introducción}

Una resolución razonable de los desacuerdos exige, entre otras cosas, que los participantes en una discusión se dejen convencer por los mejores argumentos y que abandonen los argumentos más débiles. Para ello, es necesario que exista una percepción suficientemente compartida de la calidad de los argumentos, o de qué razones apoyan qué afirmaciones. A menudo, sin embargo, esto no se da. En las discusiones cotidianas encontramos importantes desacuerdos no solo sobre afirmaciones o puntos de vista sino también en relación con la evaluación de los argumentos. Podría decirse incluso que la existencia misma de muchos desacuerdos sobre puntos de vista se debe en buena medida a que existen desacuerdos sobre la calidad de los respectivos argumentos. En tales casos, ¿cómo puede resolverse una diferencia de opinión?

Como es bien sabido, la lógica informal proporciona tres criterios básicos de evaluación argumentativa. Según fueron propuestos inicialmente, tales criterios son (Johnson y Blair, 1994, pp. 54-55):

- Suficiencia: las premisas del argumento deben proporcionar un apoyo suficiente a la conclusión.

- Pertinencia: las premisas del argumento deben ser pertinentes para la conclusión.

- Aceptabilidad: las premisas deben ser aceptables.

Con leves variaciones, o incluso con la adición de un criterio adicional-el "nivel dialéctico" en el caso de Ralph Johnson (2000)—estos criterios siguen siendo ampliamente aceptados en la actualidad. No obstante, tal como están formulados aquí, tales criterios adolecen de una vaguedad que deja mucho espacio para el desacuerdo sobre si, en un argumento determinado, se cumplen o no. No disponemos aquí de la precisión y objetividad de la noción de consecuencia lógica de los sistemas lógicos deductivos. La comprensión de los criterios de la lógica informal se apoya en gran medida en nuestras intuiciones. Esto sigue siendo cierto aun a pesar de los esfuerzos que se han realizado para esclarecer, por ejemplo, el concepto de aceptabilidad (cf. Freeman, 2005), o el concepto de pertinencia (cf. Walton, 2004).

Esta dependencia de nuestras intuiciones puede dar lugar a diferencias considerables sobre qué es aceptable, qué es pertinente y qué es suficiente. Imaginemos, por ejemplo, una discusión entre dos personas sobre si deberían reformarse las leyes relacionadas con el asesinato. Una de esas personas sostiene que deberían reformarse porque así lo han pedido los familiares de varias víctimas asesinadas; para la otra persona, en cambio, la opinión de las víctimas no es pertinente para la cuestión. $\mathrm{O}$, por mostrar un ejemplo más extremo, imaginemos una discusión con un teórico de la conspiración. Mientras que, para la mayoría de nosotros, la ausencia de pruebas de una conspiración es pertinente (o incluso suficiente) para mostrar que no hay ninguna conspiración, para el teórico de la conspiración eso muestra precisamente lo contrario: que hay una conspiración y las pruebas han sido ocultadas.

¿Cómo pueden solucionar este problema los argumentadores? Si es posible llegar a un acuerdo sobre la calidad de un argumento, parece evidente que la solución debe pasar por una metaargumentación (Marraud, 2016). Dentro de las posibilidades que ofrece la metaargumentación, en este artículo me centraré en lo que llamaré coherencia argumentativa. Mostraré cómo los argumentadores en ocasiones evalúan la coherencia de sus interlocutores y trataré de dilucidar la noción de coherencia en el ámbito de la argumentación. Tal 
como usaré aquí el término, la coherencia argumentativa no se limita a la consistencia lógica ni a una compatibilidad entre proposiciones; se trata más bien de una relación que se da entre diferentes evaluaciones de argumentos por parte de un mismo argumentador.

\section{Comprobaciones de coherencia}

Es bien conocida la anécdota que relata Bertrand Russell sobre una carta que recibió de una colega que era una lógica de renombre, en la que ella decía que era solipsista y que le sorprendía que no lo fueran también los demás (Russell, 1948, p. 196). "Viniendo de una lógica y solipsista-comenta Russell- ${ }^{1}$ su sorpresa me sorprendió.” Siendo estrictos, por supuesto, en esta anécdota no hay ninguna razón que socave el solipsismo en cuanto que postura filosófica-algo de lo que el propio Russell se dio cuenta. Sin embargo, es indudable que la defensa que la colega de Russell hace del solipsismo queda desacreditada en gran medida por la incoherencia en la que incurre.

Encontramos un caso similar en una escena de la película El gran Lebowski (1998). Un grupo de individuos que se autodenominan nihilistas exige un rescate por la liberación de una mujer que, en realidad, ellos no han secuestrado, sino que se encuentra desaparecida. Para fingir el secuestro, llegan incluso a enviar un dedo que en realidad pertenecía a la novia de uno de estos individuos. En la escena en cuestión, los nihilistas se encaran con los protagonistas, quienes ya han descubierto que no hay tal secuestro, y les piden el dinero:

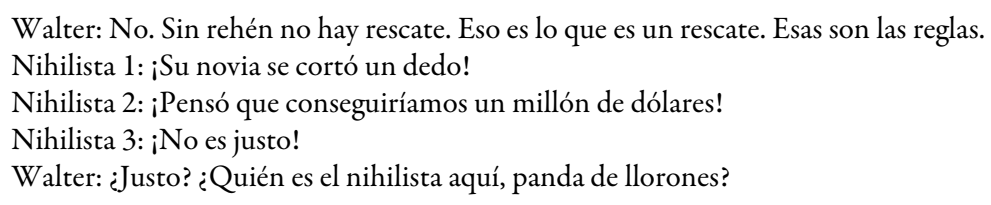

Al igual que en la anécdota de Russell, no vemos aquí ningún argumento contra el nihilismo, pero la incoherencia de estos individuos socava de algún modo su propia postura. En cada uno de estos ejemplos se da el caso de que, ante nuestros ojos, la defensa que esas personas hacen del solipsismo o del nihilismo pierde credibilidad.

En las discusiones cotidianas, especialmente cuando se trata de temas controvertidos o de posturas de difícil reconciliación, los hablantes suelen comprobar la coherencia de las opiniones de sus interlocutores. A menudo se trata de una búsqueda de incoherencias entre enunciados-por ejemplo, si alguien sostiene que mentir siempre está mal y, al mismo tiempo, que las normas de cortesía hacen que sea legítimo mentir en ocasiones-o entre enunciados y conductas. Hugo Mercier (2012) sostiene que la comprobación de la coherencia forma parte del mecanismo de vigilancia epistémica, por medio del cual evaluamos la información que otras personas nos comunican con el fin de averiguar si es fiable. Como argumenta Mercier, existen pruebas de que, cuando alguien intenta comunicarnos algo, los seres humanos comprobamos si eso es coherente con otras creencias y actitudes del comunicador.

\footnotetext{
1 En las citas de textos en inglés, la traducción es mía.
} 
Aquí, sin embargo, me centraré en un tipo de coherencia que atañe específicamente al campo de la argumentación: la coherencia entre argumentos. En la sección 4, donde detallaré con mayor precisión esta noción, explicaré con más detenimiento en qué sentido la coherencia argumentativa puede distinguirse de la coherencia entre enunciados. Pero antes, para comenzar con el análisis de la coherencia argumentativa, mostraré ejemplos de dos casos en los que se manifiesta: las acusaciones de incoherencia en lo referente a las evaluaciones argumentativas de una persona, y la búsqueda de coherencia entre un argumento que una persona ha defendido y otro argumento hipotético, que se realiza por medio de analogías.

\subsection{Acusaciones de incoherencia}

Existe cierto tipo de incoherencias que, en mi opinión, es preferible no analizar como simples incoherencias entre enunciados. Puede resultar más esclarecedor considerarlas como incoherencias argumentativas, relacionadas con la cuestión de qué razones son pertinentes o suficientes para qué puntos de vista. La coherencia argumentativa exige que, si se considera que determinadas razones apoyan una afirmación, entonces, en circunstancias similares, debe considerarse que esas mismas razones (u otras similares) apoyan una afirmación similar. De este modo, si un argumentador acepta una afirmación sobre la base de unas razones, pero no acepta otra afirmación similar que es sustentada por las mismas razones, puede ser acusado de incoherencia. Veamos un ejemplo que nos ofrece un usuario de Twitter: ${ }^{2}$

\footnotetext{
Os preocupan los manteros porque "dañan al pequeño comercio" vendiendo falsificaciones de Tous.

No como Starbucks, McDonald's, Mercadona, Carrefour, etc que no hacen ningún daño al pequeño comercio del barrio.
}

En este ejemplo se sostiene (de forma irónica) que, si los perjuicios al pequeño comercio constituyen una razón pertinente y suficiente para oponerse a la actividad de los manteros, entonces esos perjuicios también deben reconocerse como una razón pertinente y suficiente para oponerse a los grandes comercios. Si alguien acepta el primer argumento pero no acepta el segundo, incurre en una incoherencia.

A menudo, las acusaciones de incoherencia se producen porque un argumentador acepta una fuente de información en ciertos casos pero la considera poco fiable en otrosen función de si está de acuerdo con lo que afirma dicha fuente. Esto es lo que sucede en el siguiente hilo de Twitter, en el que un usuario denuncia la incoherencia con la que se recibe la información que nos proporciona la comunidad científica: ${ }^{3}$

Cuando la comunidad científica avisa sobre un riesgo, peligro, catástrofe, tiene credibilidad y es neutra*: tabaco, cambio climático...

Cuando la comunidad científica no encuentra riesgo ni peligro, carece de credibilidad y está comprada: transgénicos, glifosato, wifi...

2 https://twitter.com/Vicariusx/status/1028283413697110016

3 https://twitter.com/paleofreak/status/1028944075553206274 
${ }^{*}$ Excepto si es [sic] riesgo o peligro está en algo cotidiano que nos gusta: alcohol, exceso de azúcar, exposición al sol... entonces la comunidad científica bah, cada día dicen una cosa distinta.

(Esto pasó también con el tabaco, al principio.)

Si la comunidad científica dice algo que coincide con nuestro pensamiento, joder, es la ciencia. Si dice lo contrario, está vendida, o es patriarcal, o cuidado, que vienen otra vez los nazis, o tiene sesgos (más que tú), o sirve al sistema capitalista.

Naturalmente, no se quiere decir con esto que, si una fuente de información se considera fiable, entonces necesariamente deben aceptarse todas sus afirmaciones. Puede darse el caso de que una autoridad epistémica afirme algo que quede fuera de su campo de conocimiento o que entre en conflicto con otros conocimientos bien establecidos. Como muestran las preguntas críticas que propone Hubert Marraud para lo que él llama "argumentos doxásticos", la credibilidad de la fuente no es la única consideración que debe tenerse en cuenta para evaluar tales argumentos (Marraud, 2013, pp. 268-269):

CC1. ¿Se trata de un tema o cuestión que puede resolverse mediante una opinión cualificada?

CC2. ¿Es posible, en principio, acceder de manera directa a las razones en las que los declarantes basan su opinión?

CC3. ¿En qué se basa la atribución a $\mathrm{S}$ de la opinión C?

CC4. ¿La atribución a $\mathrm{S}$ de la creencia $\mathrm{C}$ es consistente con su conducta?

CC5. ¿Cuál es la cualificación de $S$ y cuáles las razones para atribuírsela?

CC6. ¿Qué credibilidad tiene $S$ cuando opina que C?

CC7. ¿Coincide la opinión de $\mathrm{S}$ con las demás opiniones autorizadas sobre el particular?

CC8. ¿Es $\mathrm{C}$ consistente con las pruebas materiales disponibles?

Así, aunque pueda responderse satisfactoriamente a la pregunta CC6, si las respuestas en los demás casos son insatisfactorias puede rechazarse el argumento de autoridad. Pero, en nuestro ejemplo, lo que el usuario de Twitter critica es que en determinadas ocasiones se considera que la comunidad científica "tiene credibilidad y es neutra", mientras que en otras ocasiones "carece de credibilidad y está comprada". Es decir, la incoherencia que se señala es que la respuesta a CC6 es diferente en casos distintos, a pesar de tratarse de la misma fuente.

¿Son legítimas este tipo de acusaciones de incoherencia en una discusión? Algunos teóricos tienden a pensar que no lo son. Los teóricos pragmadialécticos, por ejemplo, sostienen que las comprobaciones de coherencia deben estar restringidas a la discusión en curso (Eemeren, 2010, p. 246):

Desde un punto de vista pragmadialéctico, una inconsistencia entre algo que se dice o hace en el presente y algo que se dijo o hizo en una ocasión previa solo importa si implica una inconsistencia en una única discusión crítica.

Si la acusación de inconsistencia se refiere a dos afirmaciones que han tenido lugar en discusiones diferentes, entonces, según estos autores, tal acusación constituye una "falacia ad hominem conocida como tu quoque" (ibid., p. 248). En cambio, otros teóricos no imponen esta limitación. Walton, Reed y Macagno, al exponer el esquema del "argumento de la in- 
consistencia”, señalan que las inconsistencias "a menudo se derivan de las afirmaciones o acciones pasadas del proponente” (Walton, Reed y Macagno, 2008, p. 136). Entre las preguntas críticas que proponen para evaluar el argumento de la inconsistencia, ninguna se refiere al hecho de si la inconsistencia se produce dentro de los estrictos límites del diálogo en cuestión.

Otros autores defienden de manera explícita la importancia de la coherencia a lo largo del tiempo ${ }^{4}$. Perelman y Olbrechts-Tyteca (1989, p. 341) presentan su regla de la justicia, a la que los argumentadores apelan siempre que la coherencia de su conducta esté en entredicho. Se trata de una regla de carácter formal que prescribe "la aplicación de un tratamiento idéntico a seres o a situaciones que se integran en una misma categoría" (ibid., p. 340). Así, según esta regla, si en el pasado se ha tratado una situación de cierto modo, en el futuro debe tratarse otra situación de la misma categoría del mismo modo. En epistemología, el coherentismo que defiende BonJour (1985, p. 169) — que será comentado en la sección 3-requiere que la coherencia entre las creencias del individuo se mantenga a lo largo del tiempo para que la justificación que tal coherencia proporciona se corresponda con la verdad. Y, asimismo, en el ámbito de la argumentación jurídica, los precedentes desempeñan un papel indudablemente crucial. Como sostiene Neil MacCormick, la justificación de las decisiones jurídicas exige que estas sean universalizables, de tal modo que se traten "de igual modo los casos iguales y de diferente modo los casos diferentes" (MacCormick, 2016, p. 249). Por ello, las decisiones que se tomen en un caso determinado deben ser coherentes con las decisiones que se tomaron en casos precedentes. La noción de coherencia argumentativa que propondré en la sección 4 se apoyará precisamente en esta idea de universalización: razones similares deben conducir a puntos de vista similares, salvo que en una situación concreta existan excepciones a una regla, en cuyo caso tales excepciones también deben tomarse en consideración en otras situaciones en las que se den.

En mi opinión, las acusaciones de incoherencia que se remiten a diálogos pasados son en general legítimas. Ello se debe a que la argumentación no es un juego cerrado y autocontenido, tal como lo presenta la pragmadialéctica. Argumentamos para aumentar nuestro conocimiento, para coordinar nuestras acciones con otras personas y para saber a qué representantes políticos deberíamos votar, entre otros muchos fines. Todas estas acciones requieren cierta coherencia a lo largo del tiempo y en diálogos diferentes. El principal peligro de las acusaciones de incoherencia no es que se puedan referir a discusiones del pasado. El mayor peligro, desde mi punto de vista, reside en que a menudo los argumentadores ordinarios atribuyen a otras personas compromisos que estas no han adquirido. Esto se da especialmente cuando la acusación no va dirigida a una persona en concreto sino a todo un grupo de personas o a un colectivo-como sucede en varios de los ejemplos que hemos visto. En nuestro primer ejemplo, se asegura que quienes critican a los manteros incurren en una incoherencia porque al mismo tiempo defienden a las grandes cadenas de comercios. Pero, ¿qué pruebas hay de que todos cometan esa incoherencia? ¿Acaso no puede encontrarse a una persona que critique a los manteros y a los grandes comercios al mismo tiempo?

4 Agradezco a Paula Olmos que llamara mi atención sobre la pertinencia de la regla de la justicia para los propósitos de este artículo. 


\subsection{Analogías}

Las analogías son un tipo especial de argumentos. A menudo, los teóricos de la argumentación las han considerado como una comparación o un símil entre dos objetos o situaciones. Así lo hacen, por ejemplo, Walton, Reed y Macagno (2008, p. 56), quienes asumen que las analogías contienen la premisa "Generalmente, el caso $\mathrm{C}_{1}$ es similar al caso $\mathrm{C}_{2}$ ". En el derecho, la consideración de dos casos como análogos es la base de la argumentación a partir de precedentes. Una analogía es, según estas teorías, una comparación entre objetos o casos similares.

Sin embargo, existe otra concepción de la analogía en la argumentación —-más interesante para los propósitos de este artículo- según la cual una analogía no es una comparación entre objetos o situaciones sino entre argumentos. Entendidas de esta manera, las analogías se apoyan precisamente en la noción de coherencia argumentativa que se está proponiendo aquí. Varios teóricos han sostenido la idea de que recurrir a una analogía para defender un punto de vista implica recurrir a la metaargumentación. Al usar una analogía para argumentar, se presenta un argumento que es similar a otro en el sentido de que, si se acepta aquel, debe aceptarse también el otro. De acuerdo con Woods y Hudak (1989, p, 127):

Los argumentos de analogía son argumentos de lo que se conoce como paridad de razones. Son argumentos sobre argumentos, metaargumentos. En ellos se argumenta que dos o más argumentos diana se sostienen o se vienen abajo conjuntamente y que esto es así porque están en paridad de manera relevante, que poseen estructuras profundas similares en virtud de las cuales su forma lógica coincide.

Hubert Marraud (2007) propone un análisis similar de los argumentos que se basan en analogías. Según explica, en la argumentación por analogía se transfiere un argumento desde un campo a otro que es análogo al anterior. Quien argumenta por analogía propone "inferir la corrección de un argumento de la corrección de otro argumento", con la pretensión de que "no puede tenerse por bueno el argumento origen sin hacer lo propio con el argumento término" (ibid., p. 182).

Woods y Hudak sitúan la aparición de las analogías en un momento en el que la discusión ha alcanzado un punto muerto, cuando las razones que uno de los argumentadores presenta son consideradas como irrelevantes por el otro (Woods y Hudak, 1989, pp. 127 128). Esta es precisamente la situación que se describía en la introducción del presente artículo: cuando los argumentadores no pueden ponerse de acuerdo sobre qué es pertinente. Según Woods y Hudak, una manera de salir de ese punto muerto es recurrir a analogías.

Mi propuesta es que, en esos casos, presentar una analogía consiste en presentar un argumento que presumiblemente el interlocutor aceptará (o rechazará), con el fin de hacerle ver que, por una cuestión de coherencia, también debería aceptar otro argumento que se le ha presentado (o rechazar otro argumento que el interlocutor ha defendido). Hay, por tanto, un argumento cuya calidad está en discusión, y el argumentador presenta otro argumento que tiene una forma similar y sobre cuya calidad hay un mayor acuerdo. 5 Si la analogía es

5 No es fácil determinar qué concepción de la forma explica las analogías, pero naturalmente no se trata de la propia de la lógica formal. Aquí hablaré de "forma" y de "estructura" del argumento en un sentido manifiestamente vago. 
plausible y el interlocutor mantiene su rechazo (o defensa) del argumento, se expone a acusaciones de incoherencia.

Veamos algún ejemplo. El siguiente argumento aparece en un artículo de Scientific American sobre la hipótesis de que el cerebro humano funciona de acuerdo con el teorema de Bayes: ${ }^{6}$

Además, la tesis del cerebro bayesiano puede reducirse a un silogismo dudoso: Nuestros cerebros destacan en ciertas tareas. Los programas bayesianos destacan en tareas similares. Por lo tanto nuestros cerebros emplean programas bayesianos.

Existen unos límites obvios para esta lógica. Los halcones peregrinos destacan en el vuelo, como también los reactores F15. Nadie afirma que, por lo tanto, los halcones peregrinos deben de emplear la propulsión a chorro, porque hasta el más tonto puede ver que la mecánica del halcón peregrino y la de la propulsión a chorro son totalmente diferentes.

El autor del artículo compara dos argumentos que, según él, siguen la misma lógica. El primero de ellos es el que sostienen sus oponentes: puesto que nuestros cerebros y los programas bayesianos destacan en tareas similares, nuestros cerebros y los programas bayesianos emplean los mismos mecanismos. Al autor no le convence este argumento, presumiblemente porque las razones no son suficientes. Para mostrar a sus adversarios esta insuficiencia, lo compara con otro argumento que tiene una estructura similar y que indudablemente suscitará menos controversia: puesto que los halcones peregrinos y los reactores F15 destacan en la misma tarea (volar), los halcones peregrinos y los F15 emplean el mismo mecanismo para volar. El autor espera que sus oponentes capten la (evidente) debilidad de este argumento y ello los lleve, por una cuestión de coherencia, a concluir que el primer argumento es igualmente débil.

De este modo, en el caso de las analogías no se trata de buscar incoherencias en los argumentos del interlocutor, sino más bien de apoyarse en la coherencia con la que debe comprometerse el interlocutor si es racional. El uso de analogías argumentativas se fundamenta en una presunción de que el interlocutor es, en efecto, racional. Así que aquí juega un papel importante el principio de caridad . Este principio, como es bien sabido, fue defendido por Davidson (1973) como una condición necesaria para poder interpretar un lenguaje desconocido: de acuerdo con Davidson, debemos atribuir unas condiciones de verdad a los enunciados que intentamos interpretar tales que hagan que los hablantes estén en lo cierto en la medida de lo posible. Posteriormente fue introducido en la teoría de la argumentación como un principio de interpretación de argumentos, que Scriven (1976, p. 71) presentó de la siguiente forma: "El Principio de Caridad requiere que hagamos la mejor, y no la peor, interpretación posible del material que estamos estudiando." La aplicación del principio de caridad a la argumentación incluye la presunción de que el argumentador es racional y, por tanto, coherente. Si se le indica que existe una incoherencia en su argumentación, se espera que el argumentador la corrija. Creo que las analogías se apoyan en esta presunción: pueden convencer a un argumentador de que modifique su evaluación de un argumento por una cuestión de coherencia. Aunque, por supuesto, la presunción de coherencia es rebatible. Si el interlocutor no acepta la analogía, se expone a ser acusado de incoherencia.

6 https://blogs.scientificamerican.com/cross-check/are-brains-bayesian/

7 Agradezco a un revisor de Theoria que llamara mi atención sobre este punto. 
Veamos otro ejemplo. En The enigma of reason, Hugo Mercier y Dan Sperber hacen el siguiente comentario (Mercier y Sperber, 2017, p. 49):

La trompa del elefante es un tipo de nariz. Por muy impresionante que sea, no tendría sentido considerarla como el prototipo de las narices. De manera similar, la razón es un tipo de mecanismo de inferencia; no es el mejor ni el modelo de todos los demás.

En esta ingeniosa analogía, Mercier y Sperber se enfrentan al argumento de que, puesto que la razón es un impresionante mecanismo de inferencia, entonces debe ser considerada como el mejor y más perfecto. Para convencernos de que la capacidad de impresionarnos no es una razón suficiente para concluir que un objeto es el mejor de su clase, nos muestran un argumento análogo cuya insuficiencia todos podemos reconocer. Tal argumento sería: la trompa del elefante es un tipo de nariz impresionante, luego la trompa del elefante debe considerarse como el prototipo de las narices. Al percibir la debilidad de este argumento y la similitud entre los dos argumentos, podemos convencernos de que el argumento sobre la razón es igualmente débil.

\section{3. ¿Quées la coherencia?}

¿De qué estamos hablando exactamente cuando nos referimos a la coherencia en la argumentación? A pesar del auge de las teorías coherentistas de la justificación epistémica, de la verdad, del derecho y otras, la noción de coherencia-a diferencia de la consistencia lógica-en la bibliografía filosófica sigue siendo considerablemente vaga. ${ }^{8}$ Parece haber consenso en que la coherencia no puede reducirse a la consistencia lógica (aunque no lo hay sobre si la consistencia lógica es necesaria o no para la coherencia), pero más allá de eso existe poca claridad y poco acuerdo. Además, para los propósitos de este artículo, debe añadirse la dificultad de que aquí nos ocupamos de la coherencia en la evaluación de argumentos, mientras que la noción de coherencia que habitualmente se ha estudiado en filosofía es una relación entre enunciados. ${ }^{9}$ No obstante, con el fin de arrojar algo de luz sobre la concepción de la coherencia argumentativa que se está tratando aquí, convendrá empezar por sondear algunas de las nociones de coherencia que se han considerado en otros ámbitos.

Pueden distinguirse varios tipos de coherencia, como la explicativa, la normativa, la narrativa o la discursiva. Aquí me centraré en la coherencia explicativa y la coherencia normativa, pues son, según creo, las que más pueden ayudarnos a esclarecer la coherencia argumentativa. A continuación expondré brevemente algunos detalles de las concepciones que

8 Amaya (2015) proporciona un resumen de un amplio abanico de teorías coherentistas en epistemología, en el derecho, en la razón práctica, en ética y en la interpretación de textos.

9 En la filosofía del derecho, Soriano (2003) ha propuesto una noción de coherencia que se aplica a los argumentos que justifican las resoluciones judiciales. Sin embargo, Soriano define la coherencia sobre la base de conexiones entre las razones, de modo que no deja de ser una coherencia entre enunciados. Aquí, en cambio, busco una noción de coherencia que se aplique a diferentes evaluaciones de argumentos, así que me debo centrar en el vínculo inferencial entre razones y tesis. Además, Soriano se limita a hablar sobre la coherencia de una sola argumentación en un caso particular, mientras que para mí se trata de un concepto que se aplica también a diferentes argumentaciones en varios casos. 
proponen determinados teóricos de la coherencia explicativa y de la coherencia normativa. Me centraré en sus definiciones de coherencia y omitiré aquellos detalles de su teoría que no sean pertinentes para el propósito de explicar la coherencia argumentativa. Por razones de espacio, la exposición tendrá que ser sucinta y estar restringida a los puntos que nos interesen aquí.

En epistemología, las teorías coherentistas de la justificación epistémica constituyen una alternativa al fundacionalismo clásico. De acuerdo con estas teorías coherentistas, a grandes rasgos, una creencia está justificada si es coherente con el sistema de creencias del individuo. La noción de coherencia ocupa, por tanto, una posición crucial en estas teorías de la justificación epistémica, así que se hace necesario definirla de algún modo. Laurence BonJour (1985) reconoce que aún no hay una explicación clara y precisa del concepto de coherencia y que él mismo no la ofrece; sin embargo, proporciona ciertas condiciones orientativas de coherencia. Su punto de partida es la siguiente caracterización intuitiva de coherencia (ibid., p. 93):

Intuitivamente, la coherencia depende de lo bien que "se sostiene" un conjunto de creencias: lo bien que encajan juntas o concuerdan entre ellas las creencias que lo componen, para producir un sistema de creencias organizado, firmemente estructurado, en lugar de una recopilación atropellada o un conjunto de subsistemas en conflicto.

BonJour se centra, por tanto, en la coherencia como una propiedad de un conjunto de creencias. Las condiciones de coherencia que propone constituyen, según sostiene, un bosquejo razonable del concepto (ibid., pp. 95-99):

1. Un sistema de creencias es coherente solo si es lógicamente consistente.

2. Un sistema de creencias es coherente en proporción a su grado de consistencia probabilística.

3. La coherencia de un sistema de creencias se ve aumentada por la presencia de conexiones inferenciales entre las creencias que lo componen y se ve aumentada en proporción al número y la fuerza de tales conexiones.

4. La coherencia de un sistema de creencias se ve reducida en la medida en que esté dividido en subsistemas de creencias que carezcan relativamente de conexiones inferenciales entre ellos.

5. La coherencia de un sistema de creencias se ve reducida en proporción a la presencia de anomalías no explicadas en el contenido creído del sistema.

La condición de la consistencia lógica es, por tanto, para BonJour, una condición necesaria pero no suficiente para la coherencia. En este punto seguiremos sus pasos: creo que la consistencia, entendida en un sentido argumentativo similar al de la noción de coherencia que se busca aquí-como relación entre evaluaciones de argumentos-, podría equivaler a usar argumentos que se apoyan en principios inferenciales contradictorios. Esto, espero, se verá más claramente en la sección 4, cuando se trate la noción de regla de los argumentos. Además, a partir de la segunda condición puede verse que BonJour asume una concepción gradual de la coherencia: la consistencia probabilística absoluta no es una condición necesaria para la coherencia porque "al fin y al cabo, a veces ocurren cosas improbables" (ibid., p. 95), y las condiciones tercera a quinta establecen aumentos y reducciones de la coherencia. 
Si nos limitásemos a las dos primeras condiciones, un sistema de creencias podría ser coherente si estuviera compuesto solo por creencias totalmente independientes entre sí. Por ello son necesarias las demás condiciones, que se apoyan en la idea de relaciones inferenciales, entendidas como cualquier tipo de relación que preserve la verdad en cierto grado (ibid., p. 96). Las condiciones tercera y cuarta se apoyan en la idea de que la coherencia de un sistema de creencias será mayor cuantas más relaciones explicativas haya entre sus componentes. Por último, la quinta condición establece que un sistema de creencias resulta menos coherente en la medida en que contenga alguna anomalia, entendida como "un hecho o suceso, especialmente uno que implique algún tipo de patrón recurrente, que una o más creencias del sistema de creencias afirma que se da, pero que no puede ser explicado (o no podría haber sido previsto) apelando a las otras creencias del sistema” (ibid., p. 99).

Mi propuesta es que, para ver la pertinencia de la propuesta de BonJour para nuestra indagación sobre la coherencia argumentativa, debemos centrarnos en dos aspectos: en primer lugar, la idea de un sistema de creencias interconectadas por relaciones inferenciales (así como la idea de subsistemas independientes entre ellos); y, en segundo lugar, la idea de anomalía. Con estas dos ideas en mente, veamos las explicaciones de la coherencia que nos ofrecen otros teóricos.

Paul Thagard (2008) desarrolla una teoría de la coherencia explicativa con la que pretende explicar una diversidad de procesos de toma de decisiones, no solo en la ciencia sino también en otras áreas como el derecho. Mientras que BonJour estudiaba la coherencia de sistemas de creencias, Thagard considera que la coherencia explicativa es principalmente una relación entre dos proposiciones (ibid., p. 472). De este modo, él parte de una caracterización que toma la noción de explicación como primitiva y según la cual dos proposiciones son coherentes si hay alguna relación explicativa entre ellas - aunque admite otros tipos de relaciones de coherencia-. A continuación, Thagard propone siete principios que definen tales relaciones de coherencia explicativa. Esos principios establecen, por ejemplo, que la relación de coherencia es simétrica, que las proposiciones observacionales poseen cierta aceptabilidad intrínseca o que la inconsistencia lógica excluye la coherencia. Para los propósitos de este artículo, comentaré únicamente el principio 3 (ibid., p. 473):

\section{Principio 3. Analogía}

(a) Si $P_{1}$ explica $Q_{1}, P_{2}$ explica $Q_{2}, P_{1}$ es análogo a $P_{2}, y Q_{1}$ es análogo a $Q_{2}$, entonces $P_{1}$ y $P_{2}$ son coherentes, $\mathrm{y}_{1}$ y $\mathrm{Q}_{2}$ son coherentes.

(b) Si $P_{1}$ explica $Q_{1}, P_{2}$ explica $Q_{2}, Q_{1}$ es análogo a $Q_{2}$, pero $P_{1}$ no es análogo a $P_{2}$ entonces $P_{1}$ y $\mathrm{P}_{2}$ son incoherentes.

Este principio se apoya en las analogías, aunque se trata de analogías entre proposiciones y no de las analogías argumentativas-entre argumentos-que hemos visto en la sección anterior. El principio no establece simplemente que, si dos proposiciones son análogas, entonces son coherentes. Como explica Thagard (ibid., p. 474), la parte (a) establece que debe haber dos proposiciones análogas que expliquen respectivamente otras dos proposiciones análogas. La coherencia exige, por tanto, en este caso, una estructura explicativa. Sin embargo, Thagard se apoya en la noción de analogía entre proposiciones para explicar la coherencia, mientras que-como vimos en la sección 2.2-mi explicación aquí va en sentido contrario: se entiende la analogía argumentativa de modo que puede explicarse sobre la base de la coherencia. 
La parte (b) del principio, a su vez, establece que "cuando fenómenos similares son explicados por hipótesis desiguales, las hipótesis son incoherentes” (ibid.). Creo que esta idea puede adaptarse al ámbito argumentativo para mostrar el fundamento de algunas acusaciones de incoherencia. Puntos de vista similares deben verse apoyados por razones similares, y razones similares deben servir para apoyar puntos de vista similares. Cuando esto no sucede, por ejemplo, cuando razones similares en ámbitos distintos sirven para respaldar puntos de vista diferentes, el argumentador puede ser acusado de incoherencia.

Este punto puede relacionarse con la idea de BonJour de que la existencia de muchos subsistemas independientes de creencias reduce la coherencia. Imaginemos, por ejemplo, a alguien que argumenta que las personas de etnia uigur en China deben ser protegidas porque son una minoría, y que en otro momento argumenta que la minoría cristiana en Egipto debe adaptarse a las costumbres del país porque en este momento lo que Egipto necesita es estabilidad. Alguien podría acusar a tal argumentador de incoherencia, y el motivo es que parece estar usando criterios o reglas diferentes en circunstancias que podrían considerarse similares. Utiliza, por así decirlo, "subsistemas" de reglas argumentativas que no se relacionan entre sí. Pero aquí ya hemos pasado a hablar de reglas, lo que nos aleja de la noción epistemológica de coherencia de BonJour y Thagard y nos obliga a buscar orientación en otro ámbito: el de la coherencia normativa.

En la filosofía del derecho, MacCormick (2016) defiende la importancia de la coherencia normativa - que él distingue de la coherencia narrativa - para la justificación de un conjunto de normas jurídicas. El punto de partida de MacCormick es que la consistencia lógica es algo diferente de la coherencia, que la consistencia lógica completa no es una condición necesaria para la coherencia y que la coherencia es la propiedad que tiene un conjunto de proposiciones que, conjuntamente, "tienen sentido" (ibid., p. 321). El hecho de que un sistema de normas o una resolución judicial sean coherentes no garantiza que sean justos, pero su coherencia es una condición necesaria para que sean justificables. Una de las razones que ofrece MacCormick para defender la pertinencia de la coherencia para la justificación de un sistema de normas es que la coherencia "está en conformidad con cierta concepción de la racionalidad en la vida práctica, la que exige la universalidad y también el mayor grado posible de generalidad en los principios prácticos" (ibid., p. 338).

¿Qué quiere decir que un conjunto coherente de normas "tiene sentido"? Para explicarlo, MacCormick se apoya en las nociones de valores y de principios, que son dos caras de la misma moneda pues son extensionalmente equivalentes (ibid., p. 326):

Así, la coherencia de las normas (consideradas como algún tipo de conjunto) es una cuestión de que 'tengan sentido' al ser relacionadas racionalmente como conjunto, instrumental o intrínsecamente, con la realización de algún valor o algunos valores comunes. Esto también puede expresarse como una cuestión del cumplimiento de algún principio o algunos principios comunes articulados de manera más o menos clara. Para que los mismos principios o valores sean coherentes se requiere que puedan concebirse en su totalidad de modo que expresen una forma de vida satisfactoria.

Para comprender esta idea de coherencia como relación con unos valores o unos principios de orden superior, MacCormick nos presenta el siguiente ejemplo: "imagine una casa en la que todos los residentes tengan que desordenar sus habitaciones todo lo posible los lunes, miércoles y viernes, después ordenarlas de la manera más perfecta los martes, jueves y 
sábados, y los domingos tengan que respetarse estrictamente como día de descanso" (ibid., p. 320). Tal conjunto de normas no es inconsistente-es posible cumplirlas todas-pero ¿qué sentido tienen? ¿A qué valor o principio sirven? Son incoherentes porque no parece posible encontrar ningún principio que guíe las normas, a diferencia de, por ejemplo, las normas de tráfico que fomentan el valor de la seguridad en la carretera-entre otros.

Por último, en el ámbito de la ética, Alan Goldman (2002) defiende una postura coherentista que no se apoya en reglas o principios. Los juicios morales, sostiene Goldman, no son el resultado de subsumir un caso concreto bajo una regla. En lugar de ello, lo que se busca es la coherencia entre diferentes juicios morales, una coherencia que se basa en relaciones analógicas entre tales juicios. Los casos análogos deben juzgarse de la misma manera, y los juicios diferentes en varios casos deben apoyarse en diferencias moralmente pertinentes entre dichos casos. Esta "restricción", según Goldman, se apoya en una "interpretación caritativa parcial del principio fundamental de Kant para la razón práctica” (ibid., p. 2). Así, propone la siguiente "restricción kantiana" (Kantian constraint) para los juicios éticos (ibid., p. 161):

Como se ha indicado, esto involucra la restricción kantiana, cuya violación genera incoherencia en un conjunto de juicios morales: No se debe juzgar un caso de manera diferente de un caso previamente establecido a menos que se pueda citar una diferencia moralmente pertinente entre ellos, una diferencia que pueda mostrarse que supone una diferencia también en otros casos. Un conjunto de juicios es coherente cuando no se producen violaciones de esta restricción.

Hay, por tanto, dos partes en esta restricción: en primer lugar, debe haber alguna diferencia moralmente pertinente entre dos casos que se juzguen de manera distinta y, en segundo lugar, esa diferencia o esas diferencias deben ser aplicables de manera general también a otros casos (ibid., pp. 164-165). Así, aunque Goldman rechace la necesidad de las reglas o los principios para, en lugar de ello, apoyarse exclusivamente en las analogías, sí postula que las diferencias moralmente pertinentes deben ser generalizables. Este tipo de coherencia en la ética se justifica en parte sobre la base de la racionalidad (ibid., pp. 161-162):

Es una perogrullada decir que, si yo creo que dos contextos para la acción no pueden ser distinguidos por medio de razones para actuar de manera diferente en ellos, debo, si soy racional, actuar de la misma manera en ambos.

Un incumplimiento de esta restricción sería tratar dos casos de manera diferente, señalando alguna diferencia que se pretende pertinente pero que no puede generalizarse a otros casos. Resultaría incoherente porque se pretendería que cierto aspecto de una situación es pertinente solo en ese caso pero irrelevante en otras situaciones similares. En vista de esa falta de generalización, parece que sería difícil explicar por qué tal diferencia es pertinente e importante. Salvando las distancias, creo que en este caso de incoherencia puede ser útil el concepto de anomalía de BonJour, que vimos más arriba. Para BonJour, una anomalía era un hecho o suceso que no podía ser explicado por un sistema de creencias. Para el presente propósito de dilucidar una noción de coherencia argumentativa en la que la analogía cumple un papel importante, puede ser apropiado considerar las anomalías a partir del marco teórico de Goldman como aspectos presuntamente pertinentes que no pueden ser generalizados. 


\section{La coherencia en la argumentación}

A lo largo de este artículo he insistido en que es conveniente distinguir entre la coherencia tal como habitualmente se entiende, como una relación entre enunciados, y la coherencia argumentativa como una relación entre argumentos. ¿En qué nos ayuda esta distinción? Recordemos que, en la Introducción, la noción de coherencia argumentativa se presentaba en el marco de un desacuerdo entre dos personas sobre la calidad de un argumento. Dos de los criterios de la lógica informal para la evaluación de argumentos-la pertinencia y la suficiencia de las premisas - no se refieren a enunciados sino a relaciones entre enunciados. Un desacuerdo sobre tales criterios, por tanto, implica un desacuerdo sobre la evaluación de relaciones argumentativas entre enunciados. Así que comprobar la coherencia en la evaluación de varios argumentos no significa simplemente comprobar la coherencia entre unos enunciados: significa comprobar que premisas similares son consideradas igual de pertinentes y suficientes para conclusiones similares. En esta sección intentaré explicar más detalladamente esta idea. ${ }^{10}$

Solo tiene sentido hablar de la coherencia en la argumentación como algo deseable si aceptamos que nuestros argumentos deben fundamentarse en algún tipo de reglas generales de razonamiento. En la argumentación epistémica, la existencia de leyes y de relaciones causales es necesaria para que exista conocimiento; en la argumentación práctica, como señalaban MacCormick y Goldman, hay una concepción de la racionalidad que exige la generalidad. Las anomalías pueden entenderse, de este modo, como algún tipo de desviaciones ilegítimas de tales reglas generales. Estas consideraciones abstractas aún no nos dicen mucho sobre en qué consiste la coherencia, pero espero que, al adaptarlas a los conceptos de la teoría de la argumentación, queden un poco más claras.

Para incorporar estas consideraciones a la teoría de la argumentación, afortunadamente disponemos de un modelo de representación de los argumentos en el que tienen cabida algunos de los conceptos que se han utilizado aquí. Se trata del ya popular modelo propuesto por Stephen Toulmin. Vamos a introducir algunas de las consideraciones que hemos visto en las secciones anteriores en el modelo de Toulmin para, después, concluir esta sección con un esbozo de la noción de coherencia argumentativa.

Según el modelo de Toulmin, unas bases (o razones) apoyan una tesis por medio de una garantía, que es la que autoriza el paso de aquellas a esta. A diferencia de lo que ocurre en la lógica deductiva, el paso de las bases a la tesis no es necesario sino plausible, así que puede haber excepciones. Tales excepciones-que habitualmente se introducen con la expresión "a menos que"-pueden incorporarse en el argumento como salvedades. ${ }^{11}$ De este modo, los argumentos pueden representarse de la siguiente forma:

${ }^{10}$ Por las mismas razones, debe distinguirse entre la noción de coherencia argumentativa que se presenta aquí y la que subyace a los argumentos desde la coherencia (cf. Bertea, 2005), que sirven para argumentar a favor de un enunciado o una decisión porque es coherente con otros ya aceptados.

${ }^{11}$ El modelo de Toulmin incluye también un respaldo que fundamenta la garantía y un calificador que explicita la confianza que tiene el argumentador en la tesis. Omito estos elementos porque no desempeñan ningún papel en mi análisis de la coherencia. Véanse Toulmin (2007) y Toulmin, Rieke y Janik (2018). 


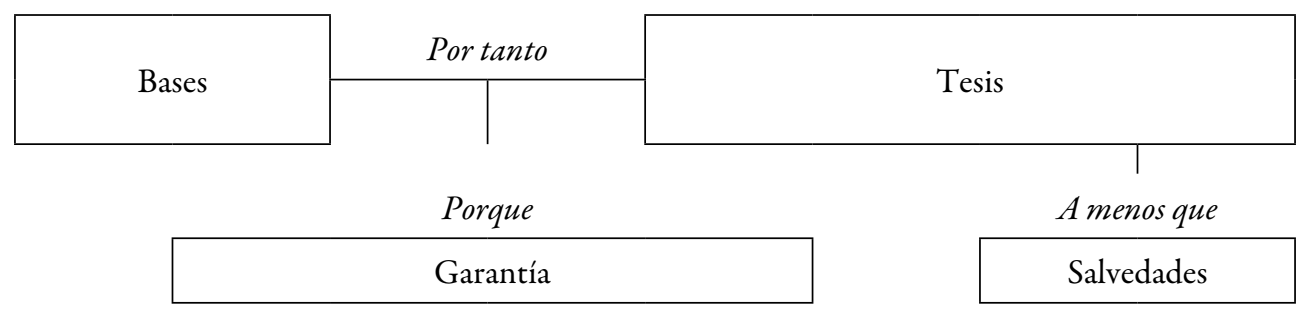

Las garantías son enunciados generales que explican el vínculo entre las bases y la tesis; o, como las presentan los propios autores, son "enunciados de unión" que "llaman la atención sobre las formas generales de argumentar” (Toulmin, Rieke y Janik, 2018, p. 79). Se trata, en resumen, de reglas generales (ibid., p. 82). Dada la generalidad de las garantías, estas deben servir no solo para sostener el argumento actual sino también otros argumentos. Si uno acepta un argumento con determinada garantía, se compromete ceteris paribus a aceptar también otros argumentos con la misma garantía. Por ejemplo, en uno de los argumentos de la sección 2.1, se denunciaba una incoherencia entre quienes en unas ocasiones se fiaban de la comunidad científica pero en otros casos afirmaban que carece de credibilidad. Ahora podemos explicar tal incoherencia como la aceptación, por parte de una misma persona, de dos argumentos con garantías incompatibles: "La comunidad científica es fiable" y "La comunidad científica carece de credibilidad".

Este caso es el más extremo de incoherencia: el que implica la aceptación de dos garantías contradictorias. Tal incoherencia puede hacerse equivaler a un caso de inconsistencia lógica. Así que, siguiendo a BonJour, podemos considerar que la coherencia exige la consistencia-no se debe aceptar una garantía y su negación en diferentes argumentos. Pero puede pensarse en casos menos flagrantes de incoherencia. Alguien podría, por ejemplo, apoyarse en las garantías de que los astrónomos son fiables pero los químicos no son fiables, o sostener que la lluvia es una buena razón para anular un partido de fútbol pero la niebla densa no lo es. Como diría MacCormick, ambos pares de reglas forman un conjunto que tiene poco sentido. No estoy seguro de si en el ámbito de la argumentación podemos apelar a una noción de "principio" similar a la que expone MacCormick en el ámbito de la filosofía del derecho, pero parece claro que las garantías de los argumentos que uno acepta deben formar conjuntos con cierto sentido.

Del mismo modo, en el caso de las analogías que vimos en la sección 2.2, lo que explica que dos argumentos análogos "se sostienen o se vienen abajo conjuntamente" es que poseen una garantía similar (Marraud, 2013, p. 200). Si la garantía se acepta en uno de los argumentos, entonces, por coherencia, debe aceptarse también la garantía en el segundo. Así, por ejemplo, en la analogía que servía para rebatir el argumento sobre el cerebro bayesiano, puede identificarse una misma garantía que subyace a los dos argumentos análogos. Recordemos que uno de los argumentos comparaba el cerebro humano con los programas bayesianos, mientras que el otro argumento comparaba los reactores F15 con los halcones peregrinos. En ambos casos se sostenía que el mecanismo debía de ser el mismo porque los objetos comparados destacaban en las mismas tareas. Por tanto, la garantía común a ambos argumentos puede formularse así: "Si dos objetos destacan en una misma tarea, emplean el mismo mecanismo." El absurdo del segundo argumento-que compara a los F15 con los halcones peregrinos-crea dudas sobre la garantía misma y en consecuencia sobre el primer argumento análogo. 
Las salvedades, por otro lado, no equivalen en absoluto a lo que antes he llamado "anomalías". La existencia de anomalías es-como indicaba BonJour-un signo de incoherencia, pero las salvedades son un elemento totalmente legítimo de los argumentos. Las salvedades son necesarias porque habitualmente las garantías no son reglas universales sino que tienen excepciones. Por ejemplo, "No se debe mentir" puede ser una garantía aceptable en un argumento, pero sin duda habrá alguna salvedad, como "A menos que lo exijan las normas de cortesía”. Lo importante es que, cuando un argumento con una garantía contenga ciertas salvedades, otros argumentos con la misma garantía también deben contener esas salvedades. Puede verse aquí la semejanza de esta idea con la restricción kantiana de Goldman, según la cual las diferencias moralmente pertinentes de una situación deben ser generalizables. El problema, lo que propongo aquí llamar "anomalía”, surge cuando se presentan en un argumento excepciones que no son generalizables a argumentos análogos.

Así que, en conclusión, podemos esbozar una caracterización de la coherencia argumentativa en términos de los conceptos de garantía y de salvedad. En la medida en que un argumentador acepte garantías y salvedades que sean generales y que, tomadas conjuntamente, sean compatibles entre ellas y formen un conjunto con sentido, el argumentador se comportará de manera más coherente. $\mathrm{Y}$ a la inversa, si un argumentador solo acepta tipos de garantías y salvedades muy específicas y que, tomadas conjuntamente, son inconsistentes o incompatibles, el argumentador será más incoherente. La coherencia aumenta en la medida en que encajen, como un todo con sentido, las garantías y salvedades que aprobamos. Por tanto, la coherencia es una noción gradual, tal como sostenía BonJour.

Esta interacción entre garantías y salvedades que fundamenta la coherencia argumentativa hace muy difícil reducir esta noción a la de coherencia entre enunciados-y por eso he insistido en distinguirlas. Lo que se busca es que las garantías que un mismo argumentador acepta sean compatibles, que las salvedades también sean compatibles y que garantías similares estén sujetas a salvedades similares. Las garantías y las salvedades son dos elementos del modelo de Toulmin que tienen que ver con la relación entre las premisas y la conclusión. Por tanto, es preferible ver la coherencia argumentativa como algo que afecta al modo en que el argumentador ve relaciones entre premisas y conclusiones.

La garantía y las salvedades dan cuenta de la pertinencia y la suficiencia de unas razones para una tesis, de modo que por medio de estos dos elementos hemos llegado al tipo de coherencia argumentativa que buscábamos. Tanto las garantías como las salvedades determinan si los datos que se presentan en las premisas son pertinentes para la conclusión; y, si lo son, si son suficientes para establecer la conclusión. Por ejemplo, si la garantía establece que la opinión de los científicos es poco fiable en cuestiones de lingüística, entonces una premisa sobre lo que ha dicho un científico respecto a la tilde en "solo" será irrelevante para una conclusión sobre si deberíamos escribir "solo" con tilde. Así que estos elementos del modelo de Toulmin nos proporcionan una herramienta para analizar las evaluaciones intuitivas de pertinencia y de suficiencia en un argumento. La coherencia en la evaluación de la pertinencia y la suficiencia en argumentos depende, en fin, de la coherencia entre las garantías y salvedades aceptadas.

El tercer criterio de la lógica informal, del que no he hablado aquí, es la aceptabilidad de las premisas. En este caso, se trata simplemente de una aceptación de enunciados, no de la aceptación de una relación entre enunciados. Por ello, el análisis de la coherencia en la aceptación de premisas resulta menos problemático. Bastará con que el argumentador no acepte razones inconsistentes o que entren en conflicto desde un punto de vista pragmático. 


\section{Conclusión}

Los criterios de la lógica informal—aceptabilidad, pertinencia y suficiencia-proporcionan un modo de evaluar la calidad de un argumento. Sin embargo, dado que la aplicación de tales criterios es en gran medida intuitiva, puede haber grandes desacuerdos entre los argumentadores sobre, por ejemplo, qué razones son pertinentes o suficientes. En este artículo he analizado un concepto que puede servir para solucionar tales desacuerdos: la coherencia. He presentado la coherencia como una relación entre evaluaciones de argumentos que se manifiesta cuando los argumentadores se acusan unos a otros de incoherencias o cuando hacen uso de analogías. A partir de ideas tomadas de teorías coherentistas en epistemología, en el derecho y en ética, he terminado por proponer un análisis basado en los conceptos de garantía y de salvedades del modelo de Toulmin.

En conclusión, si se quiere hacer uso de esta caracterización de la coherencia en un contexto pedagógico con el fin de formar a argumentadores más coherentes, el énfasis debe hacerse en la identificación de la garantía y de las salvedades, así como en la generalización de estos elementos a otros argumentos. Para evitar incoherencias, los argumentadores deben estar dispuestos a aceptar garantías y salvedades similares en argumentos análogos, y por supuesto a evitar la aceptación de garantías y salvedades inconsistentes. No existe un método sistemático e inequívoco de lograr este tipo de coherencia y, de nuevo, como ocurría con los criterios de la lógica informal, existe un margen de interpretación subjetiva en su aplicación. No obstante, como muestran los ejemplos que se han expuesto, los argumentadores de hecho comprueban si se cumple este tipo de coherencia y recurren a acusaciones de incoherencia y a analogías cuando la argumentación de primer orden-en la que se exponen directamente razones para aceptar o rechazar un punto de vista - no es suficiente para llegar a un acuerdo.

\section{REFERENCIAS}

Amaya, A. (2015). The tapestry of reason: An inquiry into the nature of coherence and its role in legal argument. Oxford: Hart Publishing.

Bertea, S. (2005). The arguments from coherence: Analysis and evaluation. Oxford Journal of Legal Studies, 25(3), 369-391.

BonJour, L. (1985). The structure of empirical knowledge. Cambridge, MA: Harvard University Press.

Davidson, D. (1973). Radical Interpretation. Dialectica, 27(3/4), 313-328.

Eemeren, F.H. van (2010). Strategic maneuvering in argumentative discourse. Amsterdam: John Benjamins Publishing Company.

Freeman, J.B. (2005). Acceptable premises: An epistemic approach to an informal logic problem. New York: Cambridge University Press.

Goldman, A.H. (2002). Practical rules. Cambridge: Cambridge University Press.

Johnson, R.H. (2000). Manifest rationality: A pragmatic theory of argument. Mahwah, NJ: Lawrence Erlbaum Associates.

Johnson, R.H. y Blair, J. A. (1994). Logical self-defense. New York: McGraw-Hill.

MacCormick, N. (2016). Retórica y Estado de Derecho: Una teoría del razonamiento jurídico. Lima: Palestra.

Marraud, H. (2007). La analogía como transferencia. Theoria 22(2), 167-188.

Marraud, H. (2013).¿Es lógic@? Análisis y evaluación de argumentos. Madrid: Cátedra.

Marraud, H. (2016). Usos y abusos de la metaargumentación. Quadripartita Ratio 1(1), 90-99. 
Mercier, H. (2012). The social functions of explicit coherence evaluation. Mind \& Society, 11(1), 81-92.

Mercier, H. y Sperber, D. (2017). The enigma of reason. Cambridge, MA: Harvard University Press.

Perelman, Ch. y Olbrechts-Tyteca, L. (1989). Tratado de la argumentación: La nueva retórica. Madrid: Gredos.

Russell, B. (1948). Human knowledge: Its scope and limits. London: George Allen \& Unwin Ltd.

Scriven, M. (1976). Reasoning. New York: McGraw-Hill.

Soriano, L.M. (2003). A modest notion of coherence in legal reasoning. A model for the European Court of Justice. Ratio Juris, 16(3), 296-323.

Thagard, P. (2008). Explanatory coherence. En J. E. Adler y L. J. Rips (Eds.), Reasoning: Studies of human inference and its foundations (pp. 471-513). New York: Cambridge University Press.

Toulmin, S. (2007). Los usos de la argumentación. Barcelona: Península.

Toulmin, S., Rieke, R. y Janik, A. (2018). Una introducción al razonamiento. Lima: Palestra.

Walton, D. (2004). Relevance in argumentation. Mahwah: Lawrence Erlbaum Associates.

Walton, D, Reed, C., y Macagno, F. (2008). Argumentation schemes. New York: Cambridge University Press.

Woods, J. y Hudak, B. (1989). By parity of reasoning. Informal Logic 11(3), 125-139.

José Ángel Gascón es doctor en filosofía por la UNED, Madrid, y actualmente becario postdoctoral CONICYT/FONDECYT en la UCSC, en Concepción, Chile. Su especialidad es la teoría de la argumentación. Ha publicado artículos relacionados con esta disciplina en Topoi, Argumentation, Informal Logic y Revista Iberoamericana de Argumentación.

Direccrón: Centro de Educación en Investigación y Desarrollo (CIEDE), UCSC, Alonso de Ribera 2850, Concepción (Chile). Email: jgascon@ucsc.cl / ORCID: 0000-0001-5571-660 